\title{
UNE METHODE DE MESURE DES SPECTRES DE PARTICULES DANS LES MILIEUX IRRADIES
}

\author{
C. HYVER* \\ (Manuscrit rȩ̣u le 6 octabre 1971)
}

\begin{abstract}
RÉSUMÉ
On expose une méthode de mesure des distributions directionnelles et énergétiques de photons ou d'électrons dans les milieux irradiés. L'objectif poursuivi est d'obtenir l'un des paramètres de base indispensables pour rendre compte de l'évolution d'un système moléculaire irradié.

Les conditions de validité expérimentale de la méthode proposée sont examinées pour le cas des photons $\gamma$.

La conclusion est que cette méthode est utilisable dans de nombreux domaines énergétiques, à condition d'y apporter les aménagements techniques nécessaires.
\end{abstract}

\begin{abstract}
A method for measuring directional and energetic distribution of photons and electrons is explained. The purpose aimed at is to obtain one of the basic parameters necessary to account for the evolution of an irradiated molecular system.

The conditions of experimental validness of the proposed method are examined in the case of $\gamma$ photons.

Our conclusion is that this method may be used in a wide range of energies, on condition that the necessary technical features of measuring facility are satisfied.
\end{abstract}

\section{INTRODUCTION}

L'étude des phénomènes élémentaires ayant lieu dans un système irradié [ $\mathrm{r}$ ] conduit à la conclusion suivante : les paramètres de base à retenir sont, d'une part, les sections efficaces d'interaction entre particules et éléments du sous-système des espèces, d'autre part, les spectres et flux particulaires qui prennent naissance dans le milieu irradié.

La détermination des spectres de particules (photons ou électrons), seule partie que nous traitons ici, peut être abordée par deux méthodes différentes mais complémentaires, par calcul ou par mesure.

La méthode par calcul n'a pas encore attiré l'attention d'un nombre suffisant de spécialistes, de sorte que ce domaine est encore mal exploré. Il est donc indispensable de vérifier, au moins au début, tout calcul à l'aide de mesures.

* Groupe d'Etudes des Effets des Rayonnements sur les Structures Moléculaires, Département de Biologie, Centre d'Etudes Nucléaires de Saclay. B.P. $\mathbf{n}^{\circ}$ 2, 91 - Gif-sur-Yvette.

RADIOPROTECTION, VOL. 7 - NO 2 
Les mesures sont du reste les seules utilisables dans le cas où la " géométrie " de la source d'irradiation et du matériau irradié est trop compliquée (problèmes de protection humaine, par exemple).

Nous voudrions proposer ici une méthode de mesure qui réponde aux objectifs suivants :

I) La méthode est susceptible de vérifier l'intermédiaire essentiel des calculs, c'est-à-dire la distribution énergétique et directionnelle des particules.

2) Elle permet de connaître la répartition énergétique du flux dans des milieux solides, quelle que soit la géométrie de l'ensemble irradiateur — milieu irradié.

\section{I - PRINCIPE DE LA MÉTHODE PROPOSÉE}

Quelle que soit la méthode utilisée, la mesure du spectre de photons ou d'électrons dans un milieu irradié introduit en général une perturbation de ce spectre.

Le problème de la mesure des spectres est donc intrinsèquement lié au problème de la connaissance ou de l'élimination des perturbations créées par le montage permettant cette mesure.

C'est en partant de cette remarque que nous proposons la méthode suivante.

Soit $(M)$ le milieu irradié, a priori de forme quelconque, et $P$ le point où l'on veut connaître les spectres de photons ou d'électrons.

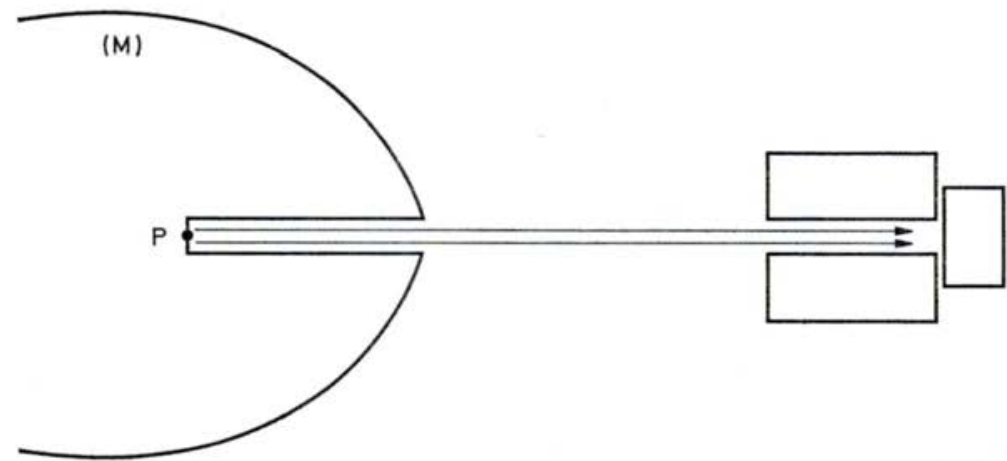

FIG. I. - Schéma de principe du montage.

L'appareil de mesure proprement dit sera placé suffisamment loin du milieu $(M)$, de façon que la présence de cet appareil ne perturbe que très peu les flux dans $(M)$. On conçoit que cette condition est relativement facile à réaliser.

Entre les deux, on réalisera le vide ou l'équivalent du vide (s'il s'agit de mesurer le rayonnement $\gamma$ dur, l'air suffira). Dans ces conditions, tout photon ou électron* émis par une paroi du milieu $(M)$ et se dirigeant vers le détecteur

* Sauf s'il s'agit d'un électron de très basse énergie (électron "thermique »). Dans ce cas, il existe un potentiel d'extraction qui invalide la proposition. 
aura une trajectoire rectiligne, aucun atome n'étant là pour l'absorber, le dévier ou dégrader son énergie.

Si l'on introduit dans le milieu un "canal " vide dirigé vers le détecteur et se terminant au point $P$, les particules émises au point $P$ pourront arriver sur le détecteur sans avoir subi de dégradation. La spectrométrie effectuée par le " détecteur» rendra alors compte du spectre au point $P$ sous trois conditions :

a) le "détecteur spectromètre " est directionnel, c'est-à-dire qu'il ne tient compte que des particules qui arrivent d'une certaine direction (celle du point $P$ );

b) le canal que l'on a creusé dans le milieu est suffisamment mince pour n'apporter qu'une perturbation négligeable;

c) le spectromètre est parfait, ou l'on sait corriger ses imperfections.

Ce sont ces trois points que nous allons examiner ci-dessous pour le domaine $\gamma$ qui présente a priori un certain nombre de difficultés.

\section{2 - LA DIRECTIVITÉ DU DÉTECTEUR}

On sait que l'appareil le plus courant permettant d'effectuer une spectrométrie $\gamma$ est le scintillateur ou le détecteur semi-conducteur. Dans ce qui suit, nous emploierons le mot scintillateur, mais il va de soi que les remarques faites s'appliquent aussi aux semicteurs.

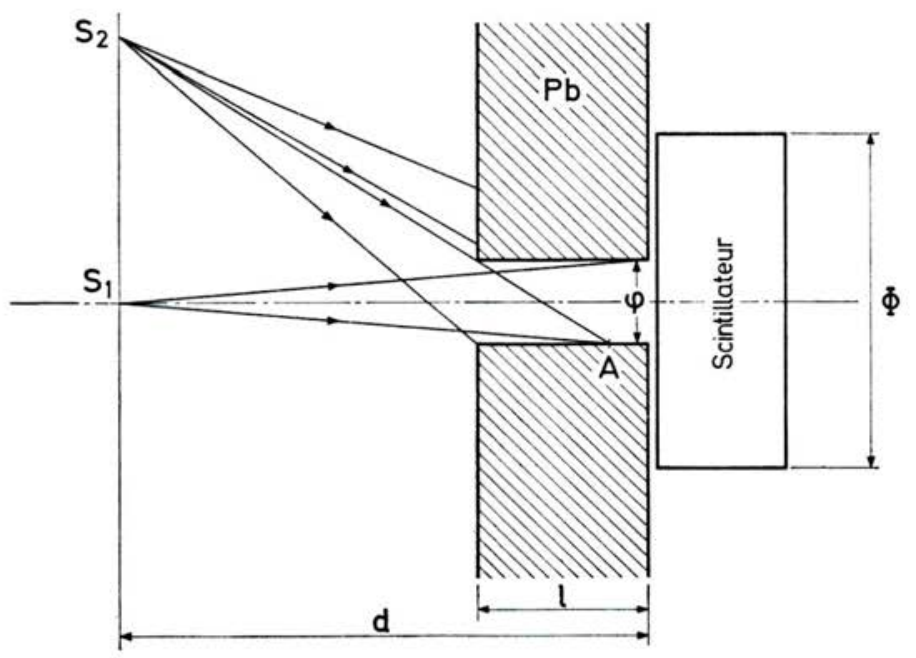

Fig. 2. - Montage directionnel en spectrométrie $\gamma$.

Dans le domaine énergétique étudié $(\approx 50 \mathrm{keV}$ à $\approx \mathrm{I}, 5 \mathrm{MeV})$, la directivité peut être obtenue simplement et à moindre frais en canalisant le faisceau du rayonnement $\gamma$ vers le centre du scintillateur à l'aide d'un blindage de plomb.

vOL. $7-\mathrm{N}^{\mathrm{O}} 2$ 
Quoique l'on puisse effectuer un certain nombre de calculs [2] susceptibles d'apporter des indications sur les performances d'un tel montage, nous ne donnerons ici que les résultats expérimentaux obtenus pour le montage défini cidessous.

\section{I. Montage expérimental}

Le spectromètre est constitué par un scintillateur ( $\left.\mathrm{I}^{\prime \prime} \frac{1}{4} \mathrm{I}^{\prime \prime}\right)$, son photomultiplicateur et l'électronique standard associée. L'enregistrement des informations s'effectue sur un 400 canaux Intertechnique SA 40 B.

Quatre types de collimateurs, de longueur $l=150 \mathrm{~mm}$, ont été utilisés; leurs diamètres étaient de $\varphi=40,8,6$ et $3,7 \mathrm{~mm}$.

La source de radiation $\left({ }^{60} \mathrm{Co}\right)$ avait les dimensions suivantes : e $=0,1 \mathrm{~mm}$, $\Phi=3 \mathrm{~mm}$.

Le schéma de principe du montage est donné à la figure 3 .

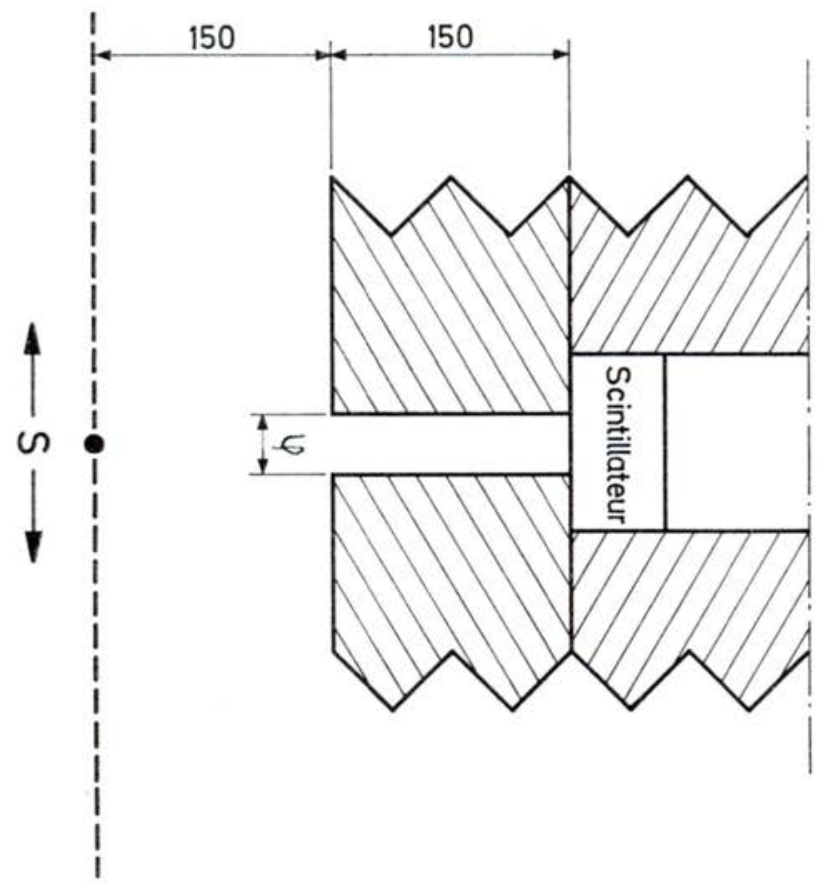

FIG. 3. - Montage de la manipulation en spectrométrie $\gamma$.

La directivité du montage s'obtient en déplaçant la source dans un plan perpendiculaire à l'axe du montage. Le relevé du taux de comptage en fonction de la distance à cet axe $(d)$ permet de tracer la courbe (fig. 4) qui donne la directivité obtenue avec un collimateur de $6 \mathrm{~mm}$ de diamètre. On remarquera que la courbe a une largeur à mi-hauteur de $12 \mathrm{~mm}$ confirmant l'ordre de grandeur de la valeur que l'on est a priori en droit d'attendre. 
MESURE DES SPECTRES DE PARTICULES EN MILIEU IRRADIE

67

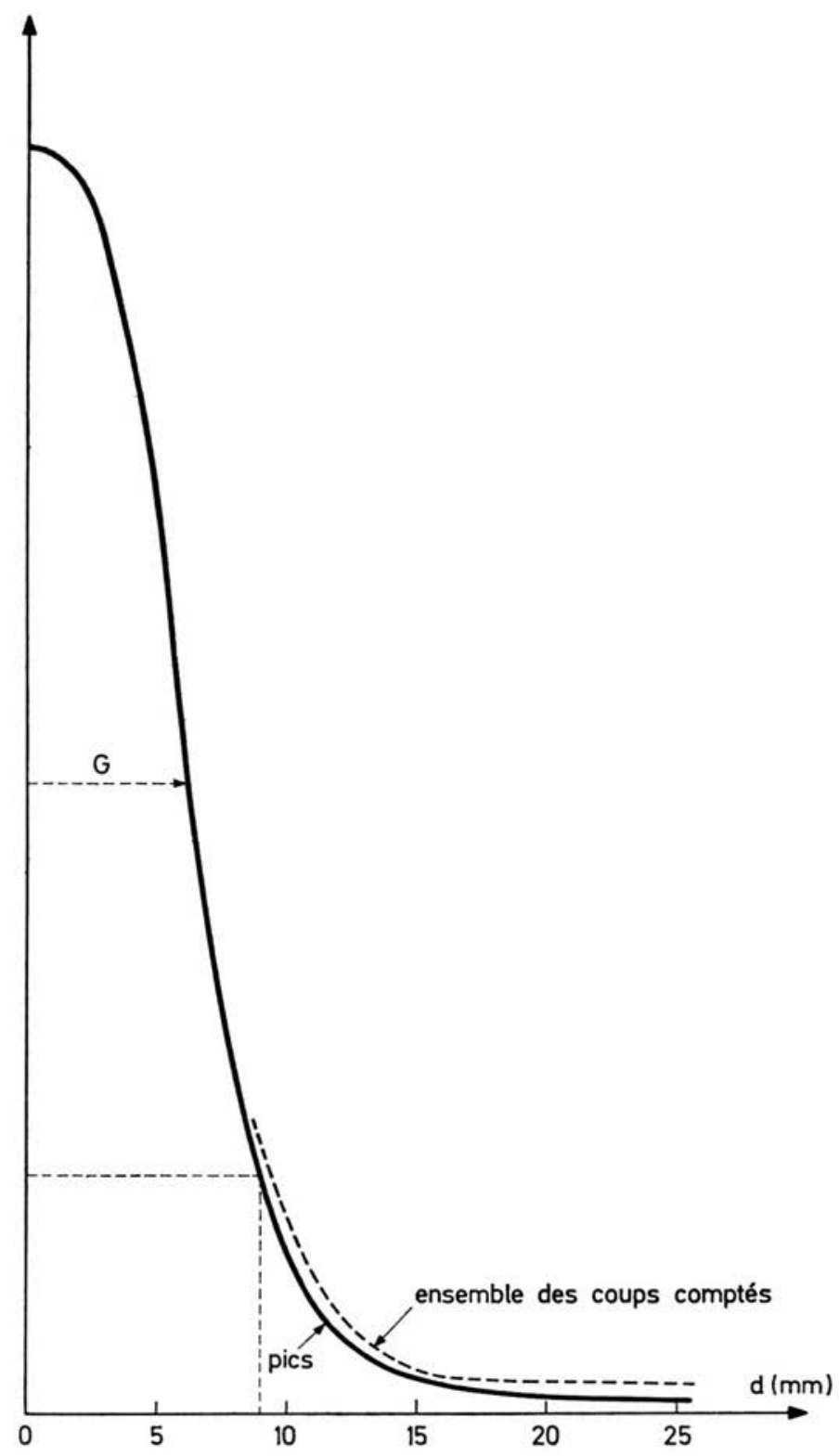

Fig. 4. - Directivité obtenue pour un collimateur de diamètre $6 \mathrm{~mm}$.

vol. $7-\mathrm{N}^{\mathrm{O}} 2$ 


\subsection{Pureté DU SPECTre D'Amplitude D'impulsions}

Le rapport $\varphi / l$ (fig. 2 ) est faible $\left(4 \cdot 10^{-2}\right.$ pour $\left.\varphi=6 \mathrm{~mm}\right)$ puisque l'on désire obtenir une bonne directivité. Le nombre de réflexions sur les parois du collimateur est alors important, et il se peut que le spectre d'amplitude d'impulsions enregistré soit sérieusement dégradé par rapport à celui que l'on obtiendrait sans collimateur.

Il est important de savoir si le spectre d'impulsions ne se dégrade pas de plus en plus lorsque $\varphi / l$ diminue. En effet, si cela était, l'analyse (voir paragraphe 4) d'un spectre d'impulsions par trop dégradé entraînerait des erreurs prohibitives sur le spectre photonique.

L'étude expérimentale de ce point a été effectuée de la façon suivante.

La source est placée sur l'axe du montage. On enregistre les spectres d'impulsions délivrées par le scintillateur pour des collimateurs de diamètres différents. Afin de pouvoir comparer facilement les enregistrements, ceux-ci ont été normalisés à même nombre de coups comptés. On a constaté, cette opération étant faite, que les courbes obtenues pour $\varphi=8, \varphi=6, \varphi=3,7 \mathrm{~mm}$ étaient identiques aux erreurs statistiques près $(\approx 5 \%)$. Toutes les erreurs statistiques sont prises, dans cette note, avec un seuil de confiance de $90 \%$ ). On a donc confondu ces courbes.

La figure $s$ représente l'enregistrement pour $\varphi=40 \mathrm{~mm}$ et celui concernant les autres collimateurs. On y voit que le spectre d'amplitude des impulsions n'est pas dégradé pour de faibles diamètres.

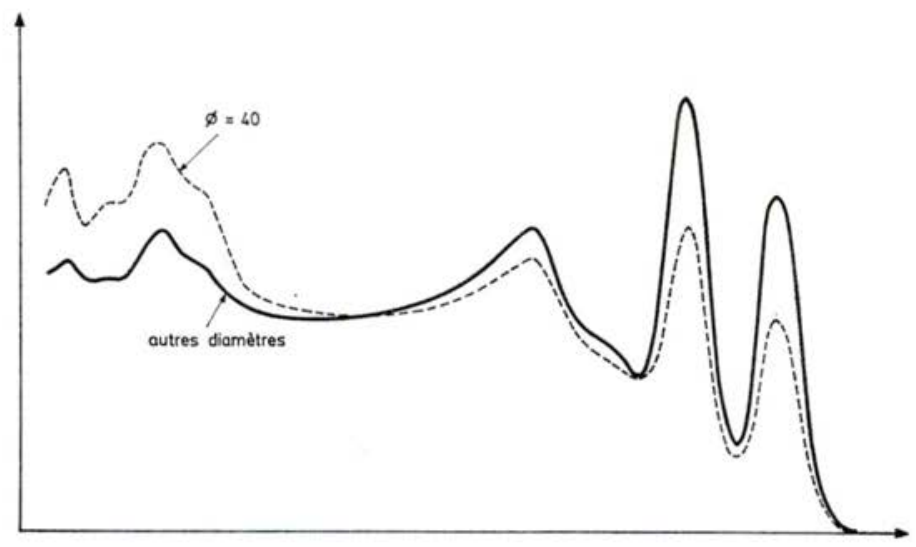

FIG. 5. - Spectres d'impulsion enregistrés pour différents collimateurs.

On conclura donc que le montage utilisé permet d'obtenir une bonne directivité sans présenter d'inconvénients susceptibles de limiter la précision des résultats que l'on recherche. 


\section{3 - PERTURBATIONS APPORTÉES PAR L'EXISTENCE DU CANAL}

Dans cette partie, nous étudions l'influence du canal que l'on a percé dans le milieu irradié. La perturbation créée par un tel canal doit être estimée, voire corrigée si besoin est. Pour ce faire, on propose d'effectuer des spectrométries avec des canaux de diamètres différents. A partir de ces données, une extrapolation à diamètre nul sera concevable sous la condition suivante : les spectres obtenus pour différents diamètres ne doivent pas être trop différents, afin que la perturbation créée par le canal ne soit pas trop grande.

Nous allons voir que cette condition est remplie pour la gamme d'énergie $\gamma$ $(\approx 50 \mathrm{keV}$ à $\approx \mathrm{I}, 5 \mathrm{MeV})$ que nous étudions, et pour une géométrie d'irradiation que nous utiliserons constamment dans la suite de cette note.

\subsection{GÉométrie et montage de L'EXpérience}

Il s'agit d'un cylindre d'altuglass de $300 \mathrm{~mm}$ de hauteur et de $300 \mathrm{~mm}$ de diamètre.

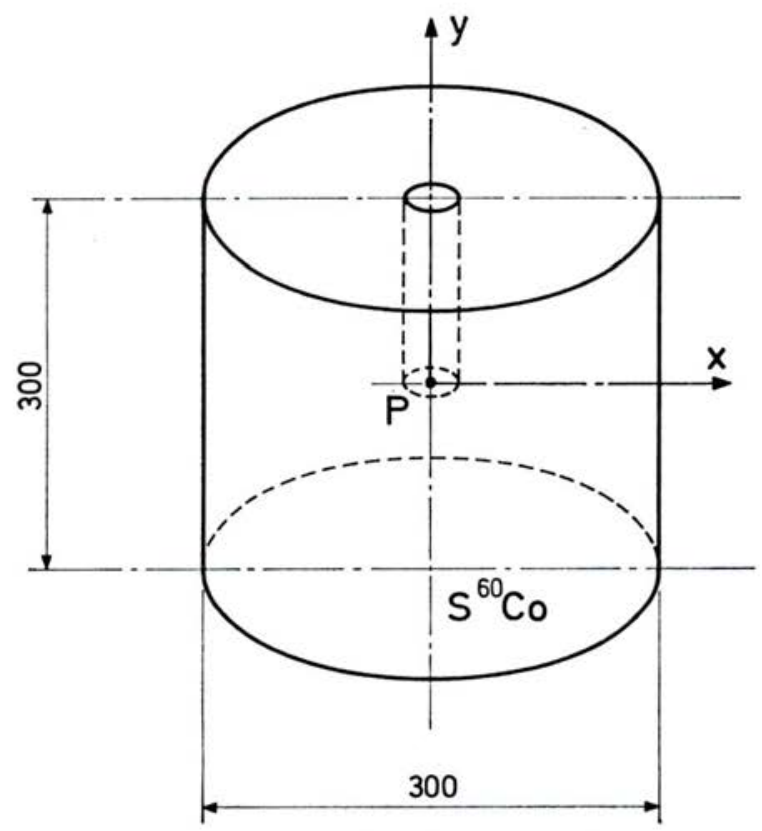

Fig. 6. - Schéma du cylindre d'altuglass.

Une source ponctuelle de ${ }^{60} \mathrm{Co}$ (de diamètre $3 \mathrm{~mm}$ et d'épaisseur $\mathrm{1} / 10 \mathrm{~mm}$ ) est située sur l'axe de révolution du cylindre.

Le point $P$ où il s'agit d'effectuer les mesures est situé sur l'axe de symétrie et à mi-hauteur du cylindre. 
Il est clair qu'en ce point, le flux n'est pas isotrope. Nous étudions ici le flux dans la sirection $\overrightarrow{S P}$. On a effectué pour cela les spectrométries avec des canaux de diamètre $\Phi^{\prime}=\mathrm{I} 2$, Is, 18 , 50 $\mathrm{mm}$.

\subsection{Résultats - Mode De DÉpouillement}

On compare ci-dessous les spectres d'amplitude d'impulsions pour les différents diamètres de canaux. Afin de ne pas rendre longue et fastidieuse la présentation des résultats ( 400 canaux $=400$ groupes de résultats), on a groupé ensemble un certain nombre de canaux de telle façon que l'on donne le nombre de coups comptés dans une certaine bande d'énergie.

On donne dans le tableau I les taux de comptage obtenus dans chacun des groupes choisis et les erreurs statistiques associées pour les différents diamètres utilisés.

TABLEAU I

\begin{tabular}{|c|c|c|c|c|c|c|c|c|c|c|}
\hline \multirow{2}{*}{\multicolumn{2}{|c|}{$\begin{array}{c}\text { Energies limites } \\
\text { bornant le groupe } \\
(\mathrm{keV})\end{array}$}} & \multirow{2}{*}{\multicolumn{2}{|c|}{$\frac{\Phi^{\prime}=12}{\text { Valeur Erreur }}$}} & \multicolumn{2}{|c|}{$\Phi^{\prime}=15$} & \multicolumn{2}{|c|}{$\Phi^{\prime}=18$} & \multicolumn{2}{|c|}{$\Phi^{\prime}=50$} & \multirow{3}{*}{ Groupe } \\
\hline & & & & \multirow{2}{*}{$\frac{\text { Valeur }}{669}$} & \multirow{2}{*}{$\frac{\text { Erreur }}{9}$} & \multirow{2}{*}{$\frac{\text { Valeur }}{639}$} & \multirow{2}{*}{$\frac{\text { Erreur }}{8}$} & \multirow{2}{*}{$\frac{\text { Valeur }}{672}$} & \multirow{2}{*}{$\frac{\text { Erreur }}{\mathrm{I}_{3}}$} & \\
\hline I 450 & 1050 & 600 & 8 & & & & & & & \\
\hline 1050 & 700 & 706 & 10 & 707 & 10 & 721 & 10 & 736 & 16 & 2 \\
\hline 700 & 430 & 509 & 10 & 514 & II & 525 & 10 & 550 & I6 & 3 \\
\hline 430 & 240 & 408 & 22 & 402 & 13 & 398 & Io & 408 & 17 & 4 \\
\hline 240 & 120 & 270 & 9 & 274 & 8 & 276 & 10 & 290 & is & 5 \\
\hline 120 & 45 & 175 & II & 185 & 7 & 178 & 9 & 177 & 12 & 6 \\
\hline
\end{tabular}

On constate que, compte tenu des erreurs expérimentales, les résultats diffèrent peu lorsque le diamètre change. Afin d'aller plus loin et d'arriver à la valeur extrapolée $\Phi^{\prime}=0$, on a fait passer par ces points, à l'aide de la méthode des moindres carrés, la courbe $N=\alpha+\beta \Phi^{\prime 2}$, cette fonction étant la plus simple que l'on puisse adopter en l'absence de renseignements théoriques précis.

Le calcul d'erreur sur la fonction d'extrapolation [2] permet de calculer l'intervalle de confiance de $\alpha$ et $\beta$. Les résultats obtenus sont consignés dans le tableau II.

\section{TABLEAU II}

VALEURS DES COEFFicients $\alpha$ ET $\beta$ DE LA COURBE $N=\alpha+\beta \Phi^{\prime 2}$

\begin{tabular}{|c|c|c|c|c|}
\hline Groupe & $\alpha$ & $\Delta \alpha$ & $\beta$ & $\Delta \beta$ \\
\hline I & 674 & 7 & $-0,62$ & 0,45 \\
\hline 2 & 708 & 8 & I, Is & 0,55 \\
\hline 3 & 512 & 8 & 1,64 & 0,55 \\
\hline 4 & 403 & II & $-0,26$ & 0,66 \\
\hline 5 & 272 & 7 & 0,75 & $0,5 \mathrm{I}$ \\
\hline 6 & $18 \mathrm{I}$ & 7 & $-0,18$ & 0,43 \\
\hline
\end{tabular}


De l'examen de ce tableau, nous tirerons la conclusion suivante : quoique les erreurs $\Delta \beta$ soient grandes, elles ne permettent pas de conclure que $\beta$ pourrait être nul. L'opération effectuée semble donc utile puisqu'elle permet de tenir compte de la difficulté due à la perturbation apportée par le canal.

\section{4 - ÉTALONNAGE \\ DU SPECTROMĖTRE DÉTECTEUR}

Il est bien connu que les effets photo-électriques, Compton... entraînent qu'une raie mono-énergétique se traduit sur l'analyseur par un spectre d'amplitudes d'impulsions relativement complexe.

Lorsqu'un spectre $\gamma$ polychromatique est stocké dans un analyseur d'amplitude, la population d'un canal donné reçoit une contribution de toutes les raies dont le pic d'absorption totale se situe dans un canal supérieur au canal considéré.

Le problème à résoudre qui consiste à déterminer le spectre réel à partir du spectre enregistré a été abordé par de nombreux chercheurs, tout au moins s'il s'agit de séparer des raies discrètes (voir par exemple [4]).

Nous allons voir maintenant la méthode d'analyse du spectre que nous avons utilisée en insistant plus particulièrement sur le problème important de la recherche de la meilleure précision possible.

\section{I. La méthode D'ANALYSE}

Les opérations à effectuer sont fondamentalement les mêmes quels que soient les auteurs.

a) Il s'agit d'abord de connaître la réponse de l'ensemble de détection pour toute raie mono-énergétique comprise dans le domaine que l'on désire explorer.

Cette opération permet de faire correspondre à un nombre de photons d'énergie $E_{i}$ issus de la source et pris conventionnellement égal à I un ensemble de valeurs $a_{i j}$ correspondant au nombre d'impulsions enregistrées dans le jième canal.

Pour franchir cette étape, nous avons utilisé une méthode empirique d'interpolation entre des spectres connus, obtenus expérimentalement [3], plutôt qu'une méthode par calcul [6]. Nous n'insisterons pas sur cette partie du travail, les programmes exposés en [3] ayant été utilisés sans aucune modification.

b) Si maintenant, un spectre incident est composé d'un ensemble de raies, le spectre enregistré sera la somme de chacune des réponses élémentaires, à condition, ce qui est notre cas, qu'il n'y ait pas empilement des impulsions.

Mais le problème inverse qui consiste à remonter de l'enregistrement au spectre qui lui a donné naissance est délicat parce qu'il est mal posé au sens de Hadamard (en fait, il admet une infinité de solutions).

Sous certaines conditions, l'équation intégrale :

$$
N(E)=\int \Delta\left(E, E^{\prime}\right) X\left(E^{\prime}\right) \mathrm{d} E^{\prime}
$$

où $N(E)$ est le nombre de coups d'énergie $E, X\left(E^{\prime}\right)$ le nombre de photons

vOL. $7-\mathrm{N}_{2}$ 
d'énergie $E^{\prime}, \Delta\left(E, E^{\prime}\right)$, la réponse du scintillateur, écrite sous la forme d'un système d'équations algébriques linéaires :

$$
N_{j}=\sum_{i} a_{i j} X_{i}
$$

$N_{j}$ : nombre de coups comptés dans le jème canal.

$X_{i}:$ nombre de photons dans la ième raie.

peut fournir une solution convenable $X_{i}=\Sigma_{i j} b_{i j} N_{j}$, $\left\|b_{i j}\right\|$ étant la matrice inverse de $\left\|a_{i j}\right\|$. Sous l'hypothèse que les erreurs sur les $N_{j}$ sont des variables aléatoires indépendantes, on a sur les variances la relation :

$$
\Delta X^{2}=\sum_{j} b_{i j}^{2} \Delta N_{j}^{2} .
$$

Telle est l'opération mathématique fondamentale qu'il s'agit d'effectuer. On trouvera en [4] [s] des exposés détaillés de cette méthode.

\subsection{Choix Des groupes Énergétiques}

Il est d'abord important de faire la remarque suivante. Il n'est pas question d'entamer un dépouillement avec des groupes d'énergie mal choisis ou trop nombreux. Si cette opération est mal effectuée, on crée explicitement ou implicitement une matrice " mal conditionnée».

Pour faire comprendre ce point, nous prendrons un exemple choisi volontairement très simple.

Soit un problème à deux inconnues $X$ et $Y$.

Il faut résoudre le système suivant :

$$
\begin{aligned}
& a X+b Y=M_{1}, \\
& c X+d Y=M_{2} .
\end{aligned}
$$

$M_{1}$ et $M_{2}$ étant les taux de comptage relevés dans les groupes I et 2. $M_{1}$ et $M_{2}$ sont entachés des erreurs statistiques $\Delta M_{1}$ et $\Delta M_{2} . a, b, c, d$ sont des coefficients dont la valeur dépend directement de la largeur énergétique des groupes.

La solution du système précédent conduit à :

$$
X=\frac{d M_{1}-b M_{2}}{a d-b c} \text {. }
$$

En supposant que les erreurs sur $M^{1}$ et $M^{2}$ soient des variables aléatoires indépendantes, on a la relation suivante entre les variantes de $X, M^{1}$ et $M^{2}$.

D'où :

$$
\Delta X^{2}=\frac{\mathrm{I}}{(a d-b c)^{2}}\left(d^{2} \Delta M_{1}^{2}+b^{2} \Delta M_{2}^{2}\right) .
$$

$$
\left(\frac{\Delta X}{X}\right)^{2}=\frac{d^{2} \Delta M_{1}^{2}+b^{2} \Delta M_{2}^{2}}{\left(d M_{1}-b M_{2}\right)^{2}} .
$$

Si le déterminant est proche de $\circ, a d \approx b c$, on aura aussi, puisque $X$ a une valeur finie, $d M_{1} \approx b M_{2}$, et l'on voit que $\frac{\Delta X}{X}$ devient très grand. 
Il est donc nécessaire, si l'on ne veut pas arriver à des erreurs prohibitives, de choisir les groupes de telle façon que $a>b$ et $d>c$; c'est-à-dire, en généralisant, de telle façon que les termes de la diagonale principale de la matrice soient propondérants vis-à-vis des autres termes.

On peut montrer [2] que cette condition conduit à la nécessité de construire les groupes d'énergie de telle façon que leur largeur $\Delta(E)$ soit proportionnelle à la résolution $\sigma(E)$ de l'appareillage, et pas trop petite vis-à-vis de celle-ci.

Cette condition peut s'expliquer d'un point de vue physique; si l'on travaille avec un appareil qui possède une mauvaise résolution, un traitement mathématique, aussi élaboré qu'on le voudra, ne saurait corriger la mauvaise résolution du détecteur.

C'est là du reste, à notre avis, que réside tout l'intérêt des semiconducteurs. Ceux-ci, possédant une résolution dix fois meilleure que les scintillateurs, permettent de présenter des résultats dix fois plus fins pour ce qui est de la variable énergie.

Les considérations précédentes expliquent pourquoi nous avons été amenés à choisir un découpage en 29 bandes d'énergie dont la largeur varie de $12 \mathrm{keV}$ aux alentours de $50 \mathrm{keV}$ à $80 \mathrm{keV}$ pour le groupe le plus élevé $(\mathrm{r}, 3 \mathrm{MeV})$.

\subsection{MÉthode de dépouillement utilisée}

Dans la méthode que nous avons utilisée, on considère que le nombre de photons existant dans un groupe énergétique donné est équivalent au nombre de photons qui seraient situés dans une raie centrée au milieu de ce groupe.

Mathématiquement, cette opération consiste à poser que les deux expressions ci-dessous sont identiques.

$$
\int_{E_{1}}^{E_{2}} \varphi(E) \Sigma(E) \mathrm{d} E=A \Sigma\left(\frac{E_{1}+E_{2}}{2}\right)
$$

$\varphi(E)$ flux différentiel.

$\Sigma(E)$ section efficace macroscopique du détecteur.

$A$ intensité de la raie située à l'énergie $\frac{E_{1}+E_{2}}{2}$.

$E_{1}$ et $E_{2}$ bornes de l'intervalle énergétique considéré.

L'examen des courbes de section efficace du corps détecteur justifie l'identification précédente.

Pratiquement, ce mode de dépouillement s'effectue de façon très simple. $\mathrm{Si}$, en effet, on a pris la précaution de situer les raies discrètes (dans notre cas, les raies du cobalt) au milieu de bandes d'énergie, il suffit de connaître les réponses du scintillateur pour les radiations monoénergétiques $\frac{E_{1}+E_{2}}{2}$ (dans notre cas, pour 29 raies) pour construire la matrice résolvante.

VOL. $7-\mathrm{N}^{\circ} 2$ 
4.4. Mesure des distributions Énergétiques et Directionnelles DU Flux DANS UN CYLINDRE D'ALTUGLASS

Nous présentons ci-dessous les résultats de mesures du flux $\gamma$ dans un milieu irradié. Il s'agit du cylindre d'altuglass que nous avons décrit au paragraphe 3.I.

Les figures 7 et 8 donnent l'histogramme des distributions mesurées au point $P$ dans les deux directions $P x$ (fig. 7) et $P y$ (fig. 8). On a regroupé les bandes d'énergie par groupes de $50 \mathrm{keV}$ (de $50 \mathrm{keV}$ à $500 \mathrm{keV}$ ) ou de $100 \mathrm{keV}$ (de $500 \mathrm{keV}$ à $\mathrm{I}, 33 \mathrm{MeV}$ ) et présenté les résultats en admettant que la densité de photons est constante dans chaque groupe. La grandeur représentée en ordonnée est le flux par unité d'énergie (échelle arbitraire).

Remarque. - Sur la direction $P y$, on mesure en fait essentiellement les deux raies de la source de ${ }^{60} \mathrm{Co}$, atténuées bien sûr par les I $5 \mathrm{~cm}$ d'altuglass traversés; la composante continue du spectre est faible et entachée d'assez grosses erreurs.

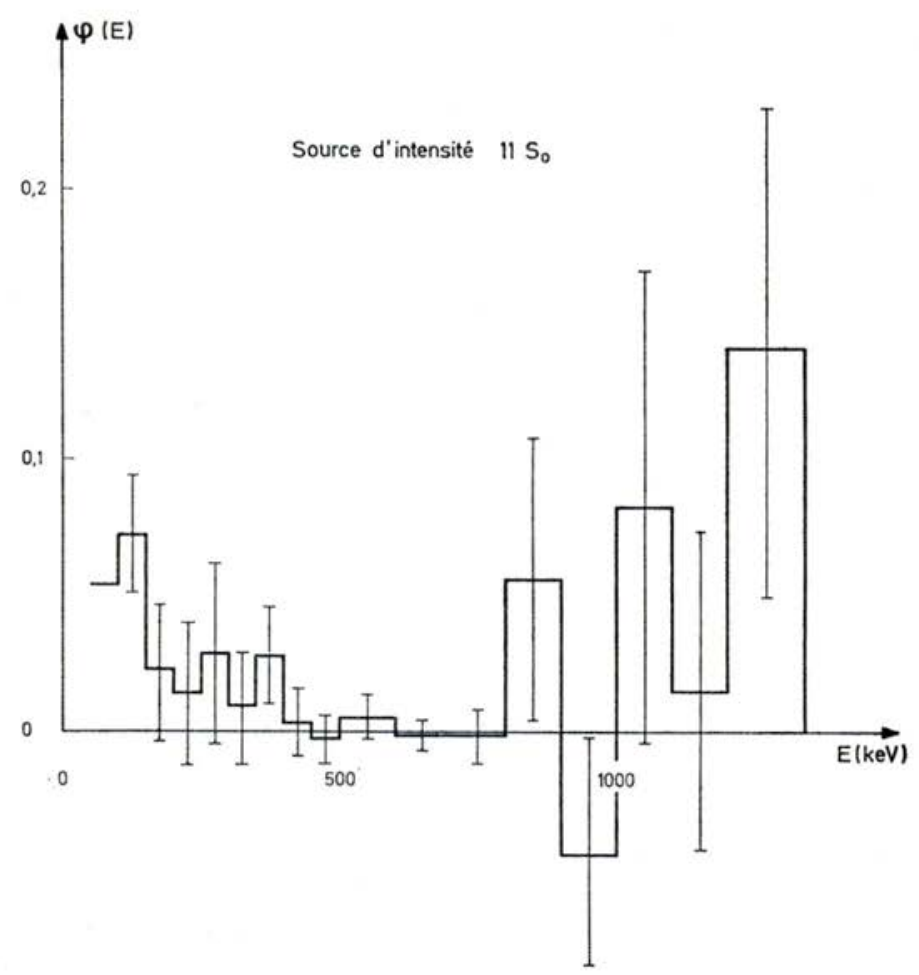

Fig. 7. - Distribution du flux dans la direction $P x$.

L'explication de ces erreurs réside dans le montage utilisé qui présente un certain nombre d'inconvénients. Nous avons déjà vu précédemment qu'un 
semicteur de bonne résolution permettrait de travailler avec des bandes d'énergie beaucoup plus faibles.

Un autre inconvénient est le suivant. A énergie assez basse, les réponses Compton provenant des énergies supérieures s'accumulent, les radiations dues à l'effet Compton étant, en effet, d'énergie plus faible que celle de la radiation initiatrice. Il est alors clair que l'extraction des flux de basse énergie est difficile et conduit à des erreurs importantes, celles-ci s'accumulant au fur et à mesure que l'on descend en énergie.

La solution qui permet de remédier à cet inconvénient, est bien connue et présente pour de tels types de mesure un avantage considérable. Il s'agit d'utiliser un dispositif " anti-Compton». On sait que de tels dispositifs sont construits précisément pour minimiser la partie Compton du spectre enregistré. On conçoit alors immédiatement le gain sur la précision aux faibles énergies que l'on peut obtenir.

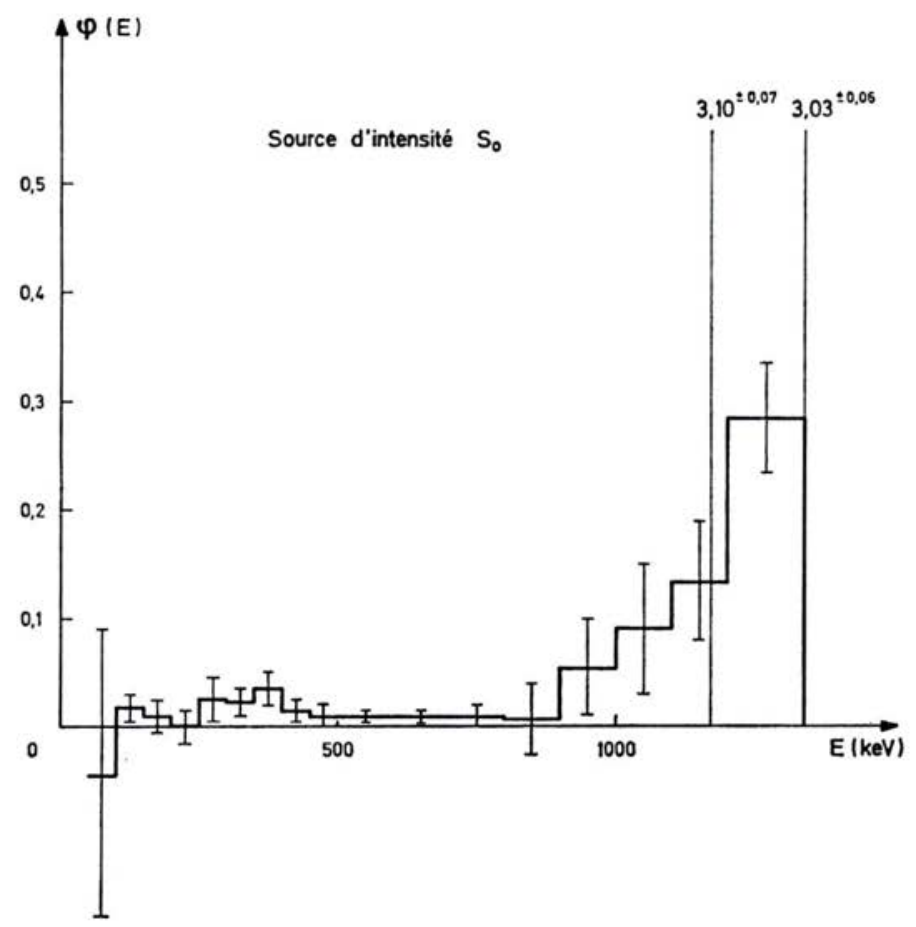

Fig. 8. - Distribution du flux dans la direction Py.

On peut donc dire que, pour les mesures que nous envisageons, le spectromètre idéal serait un semicteur muni d'un dispositif anti-Compton. 


\section{CONCLUSION}

L'étude expérimentale que nous avons décrite ci-dessus permet de conclure que des résultats satisfaisants dans le domaine $\gamma$ peuvent être obtenus à l'aide de la méthode proposée. (Dans l'état actuel de la question, il n'existe pratiquement pas de résultats théoriques ou expérimentaux de ce type).

La méthode que nous préconisons semble pouvoir s'appliquer aussi bien aux spectres d'électrons que de photons (U.V., visible par exemple). Si le principe est donc d'une grande généralité, il n'en reste pas moins que chaque gamme nécessite une étude préliminaire et la mise au point des aménagements techniques qui lui sont propres.

\section{BIBLIOGRAPHIE}

[1] Abillon E., Delatrre P., Naudet G., et Senaux R. Phénomènes élémentaires dans un système irradié. Application à la dosimétrie. Radioprotection, 1970, 5, (2), 93-1 I4 .

[2] Hyver C. Une métbode de mesure des distributions directionnelles et énergétiques des flux de particules. Rapport CEA R 4289, 1972.

[3] Boulanger J.P. Traitement des informations en spectrométrie gamma. Application à l'étude de schémas de désintégration. Rapport C.E.A. R. 3590 , 1969.

[4] Samuelli J.J., Pigneret J. et Saratin A. Instrumentation electronique en physique nucléaire, 1968, chapitre III, Masson et Cie.

[5] JACOT J. La mesure d'un rayonnement gamma par une chaîne de détection. Radioprotestion, $1969,4, n^{\circ}$ I, p. 17-4I.

[6] Zerby C.D. et Moran H.S. Calculation of the pulse height response of NaI (Tl) scintillation counters. Rapport O.R.N.L. 3169, 1962. 